\title{
Quand sexualité et religieux se croisent sur le terrain
}

\author{
Ouverture d'un chantier méthodologique
}

Céline Béraud ${ }^{1}$

Le dossier dirigé par Myriam Joël et Josselin Tricou sur «Sexualité et religion aux risques de l'enquête de terrain » est particulièrement bienvenu, tant il vient combler un vide bibliographique, du moins en langue française. Et, il est en soi significatif, qu'il soit constitué de contributions de doctorant-e.s et jeunes docteur.e-s.

Les pratiques de l'enquête ont fait en France au cours des dernières décennies l'objet d'un intérêt croissant, dont témoignent l'abondante publication de manuels et articles sur la question ainsi que les maquettes de Licence et de Master de Sociologie qui partout se sont ouvertes à ce type d'enseignement dans les années 1980.

Sur les questions de genre et de sexualité, on trouve des articles très utiles, assez récents et encore un peu épars (Buscatto, 2010 ; Montjaret, Pugeault, 2014; Schlagdenhauffen, 2014; Avanza, Fillieule, Masclet, 2015; Clair, 2016), si l'on compare aux travaux américains, en particulier aux recherches féministes qui ont développé une importante réflexion sur la place de l'enquêteur-trice dans les recherches relatives aux femmes et à l'homosexualité. La « longue hégémonie de la classe sociale » dans la sociologie française a indéniablement «formé un écran, freinant la réflexion sur l'impact du genre dans l'enquête » (Monjaret, Pugeault, 2014: 23) et contribué à la "prégnance du modèle de l'enquêteur sans sexe » (p. 29). Les grandes enquêtes sur la sexualité menées par l'INED, en particulier celle de 1992, ont certainement participé d'un tournant en la matière.

C'est la relation d'enquête qui se situe au cœur de ce corpus de textes et que l'on retrouve dans différentes contributions du dossier, qui ne s'y limite pas pour autant puisque d'autres méthodologies que celle de l'approche ethnographique y sont aussi intégrées : l'analyse quantitative (Maudet) et le travail sur archives (Deniel-Ternant). Qu'est-ce que le sexe de l'enquêteur-trice fait à l'interaction ? Le terrain, lorsqu'il s'agit de groupes monosexués, est-il plus facile lorsque l'enquêteur-trice est du même sexe que ses enquêtées ? Ne faut-il pas, au contraire, se méfier d'une complicité-proximité entre femmes ou entre hommes ? Quid de l'orientation sexuelle de l'enquêteur-trice, orientation réelle ou supposée par l'enquêté-e ? Que faut-il révéler/dévoiler ou dissimuler en la matière ? Quels effets de séduction (Clair, 2016) et de répulsion (Avanza,

\footnotetext{
1 École des hautes études en sciences sociales.
} 
2008) (Viola) ? Quels sont les risques, y compris pour son intégrité physique (Viola) et son équilibre psychique (Millet-Mouity), et quels sont les enjeux politiques et éthiques (Viola) ? Quel impact sur les données produites ?

Aucun des articles de la littérature existante, contrairement à ceux présentés dans le dossier, ne croise le religieux, à l'exception notable de la contribution de Béatrice de Gasquet (Avanza, Fillieule, Masclet, 2015). En quoi le fait de travailler sur le religieux est-il spécifique, c'est-à-dire en quoi fait-il surgir d'autres questions, d'autres difficultés ? En quoi ne l'est-il pas ? Attention, en effet, à ne pas surestimer les particularités qui seraient celles de nos objets.

Croiser religion et sexualité c'est, du point de vue de la relation d'enquête, ajouter aux dimensions déjà mentionnées celle de l'appartenance confessionnelle de l'enquêteur-trice, appartenance là aussi réelle ou supposée, présente ou passée (MilletMouity), qui suscite des assignations identitaires et nourrit des attentes du côté des enquêté-e-s. Mais cela pose aussi la question de la délimitation de l'objet et d'abord peut-être celle de la réception et de l'acceptation d'une telle recherche par les potentiel-le-s enquêté-e-s, autrement dit de la faisabilité même de telles enquêtes.

Les institutions religieuses, par les normes qu'elles édictent, ont longtemps prétendu et prétendent encore réguler les comportements sexuels de leurs fidèles, parfois même de la société dans son ensemble. Mais comment circonscrire l'objet? Qu'est-ce qu'une norme religieuse? Qu'est-ce qui relève du religieux et qu'est-ce qui relève d'autres types de normes sociales et de l'exercice d'autres formes de contrôle social ? Sur le terrain africain de Lia Viola, dans une ville à majorité musulmane, l'homophobie se dit en employant un vocabulaire religieux. L'anthropologue soutient cependant qu'il serait simpliste de considérer l'expression de cette homophobie comme étant un pur produit du religieux : « [...] à mon avis, les raisons réelles qui suscitent la violence homophobe sont à chercher dans la construction du système hétéronormatif local, auquel ne fait que participer le religieux, et non dans le système symbolique religieux lui-même » (Viola). Autre question : qu'est-ce qu'un-e enquêté-e religieux-se ? La catégorisation expose au risque de l'homogénéisation voire à celui de l'essentialisation des identités religieuses, alors que les mondes religieux sont travaillés par une grande pluralité interne et que l'appartenance confessionnelle renvoie à des liens et des engagements dont l'intensité est très variable, non seulement d'un individu à l'autre mais aussi d'un moment à un autre d'une trajectoire biographique (Gotman, 2013). Le travail sur les catégories est central pour l'analyse quantitative, comme le montre l'article de Marion Maudet qui, pour étudier les comportements sexuels des catholiques, s'est attachée à construire un indicateur de religiosité « qui ne se limite pas à l'appartenance religieuse ». L'ethnographe n'échappe pas à une réflexion sur les catégories. Vulca Fidolini est bien inspiré de ne pas définir a priori ce qu'est un musulman, par comparaison à ce que serait un profil «idéal» de fidèles, mais de s'en remettre à l'autodéfinition qu'en donnent ses enquêtés. 
Nathalie Bajos, Nathalie Beltzer et Michel Bozon considèrent que le fait d'interroger des hommes et des femmes sur leur sexualité a gagné en légitimité depuis 1992 : «la faisabilité et l'intérêt d'une enquête sur le thème n'était plus à démontrer » (2008:27). Ce serait moins le cas, dès lors que l'on croise sexualité et religion. En effet, l'autocontrôle et la prudence seraient plus forts chez les individus très intégrés à un monde religieux, qu'il s'agisse de clercs ou de fidèles zélés. Les arrangements qui ont malgré tout lieu avec la norme (Fidolini) sont dès lors plus difficiles à saisir. La peur du scandale pèse sur les institutions religieuses et leurs représentants lorsque certaines normes sont transgressées, qu'il s'agisse de "dérapages entre mineurs » ou d'actes pédophiles (Bruyère et Crosetti). Sur les questions les plus sensibles, le risque est alors de ne recueillir que des rhétoriques toutes prêtes produites par l'institution elle-même et dont les enquêté-e-s sont les porte-paroles rhétoriques qui font écran aux pratiques réellement mises en œuvre. Surtout, les questions de genre et de sexualité y sont aujourd'hui particulièrement saillantes : tant dans le débat public, dans lesquels certaines autorités religieuses et certains fidèles se sont fortement investis, que dans les controverses internes aux mondes religieux. Elles sont devenues des enjeux politiques.

L'écart entre les normes religieuses et les normes séculières relatives à la sexualité s'est creusé du fait du processus de sécularisation. Du côté du catholicisme, le célibat des prêtres, comme l'engagement à la chasteté des religieux et religieuses, constituent désormais des choix de vie atypiques voire étranges. On touche ici à l'un des points les plus nets d'illisibilité culturelle catholique, liée à «l'épuisement d'une identité qui a perdu son ancrage dans une culture commune, longtemps partagée au-delà du groupe amenuisé de ses fidèles", ce que Danièle Hervieu-Léger (2003) a qualifié d'exculturation. Les enquêté-e-s des institutions catholiques belges mettent ainsi en garde les chercheuses de l'Université libre de Bruxelles : « les sœurs religieuses ne sont pas des extraterrestres" (Bruyère et Crosetti). Mais ce décalage par rapport aux normes sociales vaut également pour les fidèles et pour d'autres mondes religieux minoritaires : par exemple, les jeunes catholiques, musulman-e-s ou protestant-e-s qui disent refuser les relations sexuelles en dehors du mariage. Les enquêté-e-s en sont parfaitement conscient-e-s et redoutent les moqueries et plus largement l'incompréhension sociale en dehors de l'entre-soi confessionnel. La crainte d'une instrumentalisation de leurs propos par les enquêté-e-s dans une perspective critique ainsi que de leur diffusion dans l'espace public, voire d'une déformation de leur discours, est très présente.

Ces dernières années, la politisation des questions de genre et de sexualité, processus auquel les groupes religieux ont contribué (Kuhar, Paternotte, 2017), renforce encore la méfiance par rapport à ce type d'enquête et plus largement à la sociologie, discipline dont on sait ce qu'elle peut «éveiller comme image négative dans un milieu fondamentalement conservateur» (Pinçon, Pinçon-Charlot, 2002). La sociologie, parce qu'elle est constructiviste, est placée du côté de la «théorie du genre » par les 
militant-e-s et ceux qui partagent leurs craintes (Bruyère et Crosetti). Elle apparaît donc comme hostile, menaçante. L'histoire et l'anthropologie ne suscitent pas la même méfiance.

Si le discours des enquêté-e-s sur la sexualité est toujours " composé » en contexte religieux et face à l'enquêteur-trice (Legrip-Randriambelo), il l'est d'autant plus qu'il est investi tant par les autorités religieuses que par les simples fidèles comme pratique distinctive. Le rappel des restrictions religieuses normatives relatives à la sexualité et au genre vise d'abord à se distinguer du monde séculier. Il fonctionne également comme un marqueur symbolique de différenciation intra-confessionnelle (Chaves, 1997; Rochefort, Sanna, 2013). À l'échelle des individus, le respect de la norme religieuse relative à la sexualité devient une performance et l'expression d'une virtuosité personnelle. C'est ainsi que les jeunes prêtres que j'interrogeais au début des années 2000 décrivaient leur rapport à la norme du célibat ecclésiastique (Béraud, 2006).

En matière de présentation de soi à l'enquêteur-trice, se joue également en contexte migratoire et d'islam minoritaire, quelque chose qui relève de ce que Vulca Fidolini appelle « l'authenticité culturelle », dont la référence à la religion est constitutive, qui se veut distinctive par rapport aux «autres», aux Européens : c'est le cas entre des hommes marocains et un chercheur italien. Le risque est alors de se laisser piéger par le processus d'essentialisation des identités ethniques et religieuses, auxquels les enquêtés peuvent eux-mêmes contribuer.

À ce stade, c'est davantage des questions qui sont soulevées par le dossier que des réponses qui sont apportées concernant les dimensions de l'enquête qui touche à la sexualité et à la religion, sujets doublement sensibles et qui ont la particularité d'être à la fois très intimes et pleinement politiques. Le dossier ouvre ainsi la voie à un vaste chantier de réflexions méthodologiques. S'il n'existe pas "d'effet pur du sexe» (Monjaret, Pugeault, 2014: 257), pas davantage d'ailleurs que du religieux, on observe ici en quoi la prise en compte de la sexualité et du genre produit un effet de levier, certainement accru par son croisement avec le religieux, pour penser les situations d'enquête.

\section{Bibliographie}

Avanza M. (2008), "Comment faire de l'ethnographie quand on n'aime pas "ses indigènes" ? Une enquête au sein d'un mouvement xénophobe », in D. FASSIN, A. BENSA (dir.), Les politiques de l'enquête. Epreuves ethnographiques, Paris, La Découverte, p. 41-58.

Avanza M., Fillieule O., Masclet C. (2015), « Ethnographie du genre. Petit détour par les cuisines et suggestions d'accompagnement. Introduction du Dossier », SociologieS. 
Bajos N., Beltzer N., Bozon M. (2008), «Enjeux méthodologiques et recueil de données », in N. BAjos, M. Bozon (dir.), Enquêtes sur la sexualité en France. Pratiques, genre et santé, Paris, La Découverte, p. 27-44.

BERAUD C. (2006), «Prêtres de la génération Jean-Paul II : recomposition de l'idéal sacerdotal et accomplissement de soi ", Archives de sciences sociales des religions, vol. 133, p. 45-66.

Buscatto M. (2010), La fabrique de l'ethnographe. Dans les rouages du travail organisé, Paris, Octarès.

Chaves M. (1999), Ordaining Women: Culture and Conflict in Religious Organizations, Cambridge, Harvard University Press.

Clair I. (2016), «La sexualité dans la relation d'enquête. Décryptage d'un tabou méthodologique ", Revue française de sociologie, vol. 57, p. 45-70.

GASQUET B. DE (2015), «Que fait le féminisme à l'ethnographe ? La réflexivité sur le genre comme connaissance située », SociologieS.

Gotman A. (2013), Ce que la religion fait aux gens, Paris, Éditions de la Maison des Sciences de l'Homme.

Kuhar R., Paternotte D. (dir.) (2017), Anti-Gender Campaigns in Europe. Mobilizing against Equality, Londres, Rowman \& Littlefield International.

Monjaret A., Pugeault C. (dir.) (2014), Le sexe de l'enquête. Approches sociologiques et anthropologiques, Lyon, ENS Éditions.

Pinçon M., Pinçon-Charlot M. (2002 [1997]), Voyage en grande bourgeoisie. Journal d'enquête, Paris, Presses universitaires de France ("Quadrige»).

ROCHEFORT F., SANNA M.-E. (dir.) (2013), Normes religieuses et genre. Mutations, résistances et reconfiguration $\mathrm{XIX}^{e}-\mathrm{XX} \mathrm{X}^{e}$ siècle, Paris, Armand Colin.

SChlagdenhauffen R. (2014), «Parler de sexualité en entretien. Comment rendre publics des propos privés ", Hermès. La Revue, n 69, p. 34-38. 